\title{
Concurrent triplication and uniparental isodisomy: evidence for microhomology-mediated break-induced replication model for genomic rearrangements
}

\author{
Trilochan Sahoo ${ }^{\star 1}$, Jia-Chi Wang ${ }^{1}$, Mohamed M Elnaggar ${ }^{1}$, Pedro Sanchez-Lara ${ }^{2}$, Leslie P Ross ${ }^{1}$, \\ Loretta W Mahon ${ }^{1}$, Katayoun Hafezi ${ }^{1}$, Abigail Deming ${ }^{1}$, Lynne Hinman ${ }^{1}$, Yovana Bruno ${ }^{3}$, James A Bartley ${ }^{2}$, \\ Thomas Liehr ${ }^{4}$, Arturo Anguiano ${ }^{1}$ and Marilyn Jones ${ }^{5,6}$
}

Whole-genome oligonucleotide single-nucleotide polymorphism (oligo-SNP) arrays enable simultaneous interrogation of copy number variations (CNVs), copy neutral regions of homozygosity (ROH) and uniparental disomy (UPD). Structural variation in the human genome contributes significantly to genetic variation, and often has deleterious effects leading to disease causation. Co-occurrence of CNV and regions of allelic homozygosity in tandem involving the same chromosomal arm are extremely rare. Replication-based mechanisms such as microhomology-mediated break-induced replication (MMBIR) are recent models predicted to induce structural rearrangements and gene dosage aberrations; however, supportive evidence in humans for oneended DNA break repair coupled with MMBIR giving rise to interstitial copy number gains and distal loss of heterozygosity has not been documented. We report on the identification and characterization of two cases with interstitial triplication followed by uniparental isodisomy (isoUPD) for remainder of the chromosomal arm. Case 1 has a triplication at 9q21.11-q21.33 and segmental paternal isoUPD for 9q21.33-qter, and presented with citrullinemia with a homozygous mutation in the argininosuccinate synthetase gene (ASS1 at 9q34.1). Case 2 has a triplication at 22q12.1-q12.2 and segmental maternal isoUPD 22q12.2-qter, and presented with hearing loss, mild dysmorphic features and bilateral iris coloboma. Interstitial triplication coupled with distal segmental isoUPD is a novel finding that provides human evidence for one-ended DNA break and replication-mediated repair. Both copy number gains and isoUPD may contribute to the phenotype. Significantly, these cases represent the first detailed genomic analysis that provides support for a MMBIR mechanism inducing copy number gains and segmental isoUPD in tandem.

European Journal of Human Genetics (2015) 23,61-66; doi:10.1038/ejhg.2014.53; published online 9 April 2014

\section{INTRODUCTION}

One of the most significant outcomes of clinical implementation of high-resolution genome-wide microarrays is the discovery of rare complex chromosomal rearrangements (CCRs). The addition of single-nucleotide polymorphism (SNP) probes in microarrays has the added advantage of enabling identification of long contiguous regions of allelic homozygosity $(\mathrm{ROH}) \cdot{ }^{1-3}$ These copy neutral aberrations are either multiple across the genome and likely due to identity by descent (IBD), or due to segmental or whole chromosome uniparental disomy (UPD) when restricted to a single chromosome. The associated findings of a cytogenetic abnormality with whole chromosome uniparental disomy is well known and, in some cases, a recurrent phenomenon. This simultaneous occurrence of both abnormalities is particularly common when a marker chromosome, whole chromosome aneuploidy (often mosaic) or Robertsonian translocation, is observed. ${ }^{4-6}$ It has been suggested that more than a third of cases of UPD are the likely outcome of or are associated with a chromosomal abnormality. ${ }^{6}$ Therefore, the chromosomal contribution to UPD formation is a more common phenomenon than commonly perceived. Formation of whole chromosome UPD or a variety of segmental UPD as a consequence of precedent chromosomal rearrangements reflects both meiotic and mitotic recombination events likely being involved.

Genomic triplications are rare unbalanced chromosomal aberrations with variable clinical effects. ${ }^{7-10}$ The association of triplication and segmental uniparental isodisomy (isoUPD) has been reported in only one single case. ${ }^{8}$ Deciphering the mechanism of triplications has remained challenging because of the rarity of the events and the difficulty in defining the breakpoints with accuracy. It has been recently proposed that triplications evolve following one of a number of pathways including nonallelic homologous recombination (NAHR), break-induced replication (BIR), fork stalling and template switching (FoSTES) or microhomology-mediated BIR (MMBIR). ${ }^{11-15}$ In contrast to triplication, CCRs typically involve multiple chromosomes with multiple breakpoints and are usually nonrecurrent. ${ }^{16-18}$ The co-occurrence of an interstitial triplication contiguous distally with segmental isoUPD introduces a new level of complexity to our understanding of the cause, mechanism and phenotypic effect of chromosomal rearrangements. In addition to the genomic burden of the dosage effect because of the triplication, the concurrent $\mathrm{ROH}$ introduces segmental uniparental isodisomy and unraveling of homozygosity for recessive disease mutations. Here we

${ }^{1}$ Quest Diagnostics Nichols Institute, San Juan Capistrano, CA, USA; ${ }^{2}$ Children's Hospital Los Angeles, Division of Medical Genetics, Los Angeles, CA, USA; ${ }^{3}$ White Memorial Medical Center, Los Angeles, CA, USA; ${ }^{4}$ Institut für Humangenetik, Jena, Germany; ${ }^{5}$ Rady Children's Hospital, Division of Genetics/Dysmorphology, San Diego, CA, USA; EUniversity of California, San Diego, CA, USA

*Correspondence: Dr T Sahoo, Quest Diagnostics Nichols Institute, 33608 Ortega Highway, San Juan Capistrano, CA 92690 , USA. Tel: +1 949 728 4760; Fax: +1 949728 4979; E-mail: trilochan.x.sahoo@questdiagnostics.com

Received 17 September 2013; revised 17 February 2014; accepted 19 February 2014; published online 9 April 2014 
report detailed clinical and molecular characterization of two cases with interstitial triplications and segmental uniparental isodisomy. Both cases, including additional cases not exhaustively characterized, provide an exciting new frontier, expanding the scope of our understanding of the nature, structure and phenotypic effects of chromosomal rearrangements.

\section{PATIENTS AND METHODS}

Appropriate institutional IRB-approved consents was obtained from patient's families for detailed follow-up studies for publication purposes.

\section{Case 1}

This patient is a 5-year-old girl with a known clinical diagnosis of citrullinemia, neuro-developmental problems and a chromosome 9 abnormality. Pregnancy was complicated by uncertain dating and decreased fetal activity. Birth weight at this examination was $1.81 \mathrm{~kg}$. Dysmorphic features were not appreciated at birth. She presented with lethargy coincident with the suggestion on newborn screening of a urea cycle defect. In early infancy, she had mild episodes of hyperammonemia, which were easily managed; however at age 2 she had a severe episode of hyperammonemia with cerebral edema. Sitting, walking and talking are mildly delayed. She survived but has developed severe spastic quadreparesis and intellectual disability. She has subsequently had a liver transplant, which has stabilized her metabolic condition. At age 5, her examination revealed microcephaly and a severely growth retarded girl with spastic quadreparesis, cortical blindness and hearing loss. She has downslanting palpebral fissures, a pointed nasal tip, severe micro/retrognathia, hypoplastic labia majora and minora, many disuse creases on her hands and areas of hypo- and hyperpigmentation in her skin. Laboratory studies showed the levels of argininosuccinate synthetase (ASS) are unmeasurable.

\section{Case 2}

This patient is a $39 / 12$-year-old girl. Her birth weight was $2.27 \mathrm{~kg}$, and she was microcephalic. At 3 years of age, she was at the 10th centile for both weight and height, with head circumference less than the $3 \mathrm{rd}$ centile $(45.8 \mathrm{~cm})$. She has dysmorphic facies, is speech delayed and has intellectual disability. She receives speech and language therapy. She also has intermittent conductive hearing loss, but her sensori-neural hearing is normal. Her ear helices are short for age and the superior helix is thickened. The irises each have a coloboma inferiorly. Her nasal bridge is flat. The labia minora is hypoplastic. Her joints are mildly lax and she has decreased muscle tone. An initial consideration was CHARGE association.

Oligonucleotide-SNP (oligo-SNP) microarray analysis. The above two cases were identified from over 14500 cases analyzed utilizing the Affymetrix version 6.0 genechip ( $\sim 5700$ cases; Affy 6.0 includes $>900 \mathrm{k}$ non-polymorphic copy number probes and $>900 \mathrm{k}$ SNPs), or the Affymetrix CytoScan HD Array (8800 cases; whole-genome $2.7 \mathrm{M}$ array) (Affymetrix, Santa Clara, CA, USA). Our analysis used thresholds of $50 \mathrm{~kb}$ for losses and $200 \mathrm{~kb}$ for gains for the whole genome. Lower thresholds ( $20 \mathrm{~kb}$ for losses and $100 \mathrm{~kb}$ for gains) were used for $>250$ cytogenetic relevant genomic intervals. The threshold for ROH/ UPD were set at $5 \mathrm{Mb}$ with summation provided for all segments $>5 \mathrm{Mb}$. Uniparental disomy is considered upon identification of a single segment of $\mathrm{ROH}>10 \mathrm{Mb}$ or, two or more segments $>5 \mathrm{Mb}$ each involving a single chromosome. The Affymetrix Chromosome Analysis Suite Software (ChAS; NetAffx Version 32; Affymetrix) was used for data analysis and review. Genomic coordinates are based upon genome build 37/hg19.

Chromosome karyotype and FISH analysis. The peripheral blood (Na-heparin) was cultured for $72 \mathrm{~h}$ in RPMI-1640 medium (with FBS and L-glutamine-penicillin-streptomycin). Metaphase chromosomes were analyzed by standard GTG-banding techniques. Fluorescence in situ hybridization (FISH) analysis was performed with the BAC clones physically mapped to within the abnormal segments (Figures $1 \mathrm{~b}$ and $2 \mathrm{~b}$ ). Genomic coordinates for BAC clones are based upon genome build 37/hg19 (case 1: CTD-2059K7, red, GRCh37/hg19: Chr9: 71599589-71746937; and RP11-522I20, green, GRCh37/hg19: Chr9: 86 185 715-86354 418) at the centromeric and telomeric end of the triplicated segment, respectively; case 2: (RP11-419L7, green, GRCh37/hg19: Chr22: 28122 228-28304019; and, CTD-2010F20, red, GRCh37/hg19: Chr22: 9647 821-29759207).

\section{RESULTS}

\section{Oligo-SNP array analysis}

Among the over 14500 samples analyzed by oligo-SNP array in the laboratory, the detection rate for clinically significant copy number variations (CNVs) was 10\% (J-C Wang et al (2013), personal communication). CNVs of unclear clinical significance were identified in $22 \%$ of cases. Approximately 832 (6\% of all cases analyzed) cases from 801 families harbored $\mathrm{ROH}$, with 651 cases (78\%) harboring multiple $\mathrm{ROH}$ likely due to IBD or varying degrees of consanguinity (IBD), and 181 cases $(22 \%)$ cases with $\mathrm{ROH}$ involving a single chromosome or chromosomal segment (J-C Wang et al (2013), personal communication). In our testing population, cases with UPD were statistically more likely to harbor a clinically significant $\mathrm{CNV}$ as compared with the entire testing population $(P<0.001)$.

\section{Case 1}

Microarray analysis in case 1 revealed two additional copies (triplication) of a 16-Mb segment of the proximal long arm of chromosome 9 (9q21.11-q21.33). In addition, the SNP data revealed homozygosity for entire $9 \mathrm{q}$ immediately distal to the triplicated segment $(9 \mathrm{q} 21.33$ q34.3; $54 \mathrm{Mb}$ ) (ISCN: $\operatorname{arr}[\mathrm{hg} 19]$ 9q21.11q21.33 (70 984 588$86956612) \times 4, \quad 9 q 21.33 q 34.3 \quad(86956612-140955352) \times 2 \quad \mathrm{hmz})$ (Figure 1a). Therefore, the triplicated segment and the isodisomic segment share a breakpoint at $9 \mathrm{q} 21.33$. Genotype analysis for all informative SNPs within the isodisomic segment for this case and both her parents revealed that the isodisomic segment was paternal in origin. As can be seen from the 'Allele peaks' track (Figure 1a), the triplicated segment showed three tracks reflective of AA/AA $(+2)$, $\mathrm{AB} / \mathrm{AB}$ or $\mathrm{AA} / \mathrm{BB}(0)$ and $\mathrm{BB} / \mathrm{BB}(-2)$. The most likely possibility of the heterozygous $\mathrm{AB} / \mathrm{AB}$ showing a value equivalent to $\mathrm{AB}(0)$ is due to equivalent contribution of this segment from both parents (two copies maternal and two copies paternal). This conclusion is complemented by the fact that for SNPs (within triplicated segment) where parents are homozygous for alternate alleles (AA in one parent and $\mathrm{BB}$ in one parent, and vice versa), the proband reflected a heterozygous $\mathrm{AA} / \mathrm{BB}$ (' 0 ' or $\mathrm{AB}$ ) pattern.

Karyotype and FISH analyses. The initial cytogenetic analysis revealed a abnormal female karyotype of 46,XX,dup(9)(q12q22). Preliminary FISH analysis by whole chromosome 9 painting probe confirmed the extra segment was derived from chromosome 9 $(46, X X, \operatorname{dup}(9)(q 12 q 22)$.ish $\operatorname{dup}(9)(w c p 9+))$. Dual-color FISH analysis utilizing two BAC clones at the two opposing ends of the segment provided some clarity of the arrangement (Figure 1b). Extended FISH analysis (evaluation of at least 200 interphase nuclei and 50 metaphase cells) ruled out mosaicism for the copy number alteration as no normal metaphase or interphase cells were detected. A diagrammatic representation of the arrangement of triplicated segment is shown in Figure 1c. The likely maternal and paternal segments contributing to the triplication are indicated (by pink and blue arrows for maternal and paternal segments, respectively). Cumulative data from microarray analysis, FISH and G-banded chromosome karyotype analysis (data not shown) revealed a direct-inverted-direct conformation for the triplicated segment that apparently includes most of the $9 \mathrm{q}$ pericentromeric heterochromatin (9qh) (Figure 1, panels b and c). 


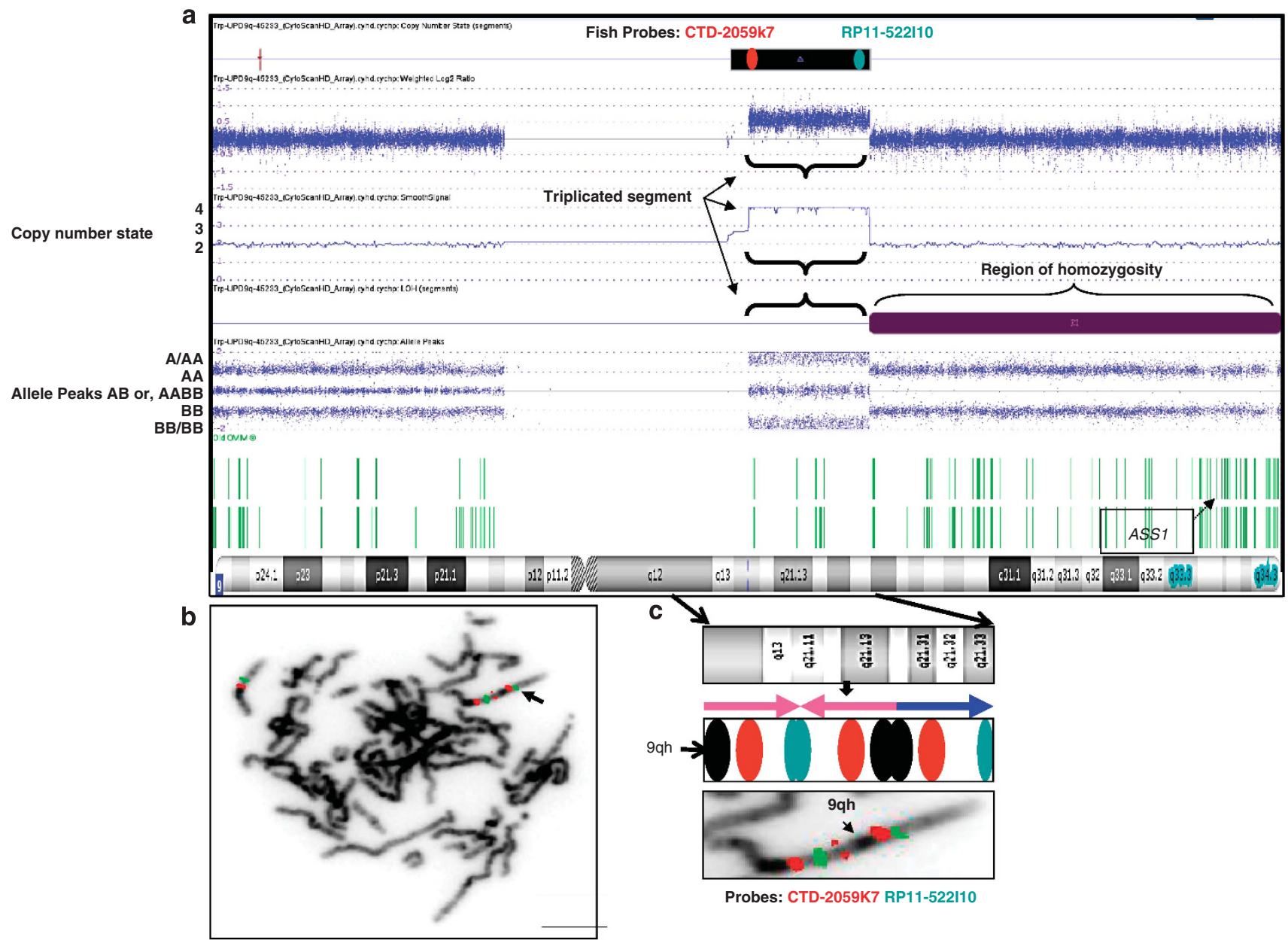

Figure 1 Panel a, case 1. Copy number Log2 ratio, copy number state and allele peak plots for case 1 . Segmental triplication (copy number state $=4$ ) indicated by horizontal brackets; location of BAC clones used as FISH probes are schematically represented as red and green ovals within the triplicated segment (CTD-2059K7, red; RP11-522I20, green; GRCh37/hg19 coordinates described in Patients and methods section). Allele peak plot across the triplicated segment reflects wider dispersion of $A A / A A$ and $B B / B B$ alleles (four copies) whereas the $A A B B / A B A B$ alleles plotting identical to heterozygous $A B$ alleles; and loss of $A B$ heterozygous genotype in the distal isodisomic segment. Panels (b, $\mathbf{c}$ ) show FISH confirmation of interstitial triplication and putative arrangement of the segment in a direct-inverted-direct orientation (panel c) (9qh refers to the chromosome 9 pericentromeric heterochromatin segment that is apparently partially involved in the triplication). Panel c shows magnified segment (9qh-q21.33) that is involved in the triplication. Bottom half of panel $\mathbf{c}$ is schematic representation of the likely organization of the triplication segment; pink arrow reflects maternal origin (two inverted segments) and blue arrow reflects paternal origin (direct orientation). Orientation of the triplicated segment that apparently includes a part of the pericentromeric heterochromatin is derived from the signal pattern ( $1 x$ red $-2 x$ green $-1 x$ red- $2 x$ partial heterochromatin segment- $1 x$ red $-1 x$ green).

The triplicated segment includes 44 OMIM annotated genes, 7 of which have been associated with a clinical phenotype (Supplementary Table 1). None of the seven described OMIM annotated disorders were considered as contributing significantly toward the patients phenotype. The distal homozygous segment includes 342 OMIM annotated genes including ASS1 (OMIM 603470) (Supplementary Table 1). The cause of autosomal recessive citrullinemia (OMIM 215700, ASS1 gene at $9 \mathrm{q} 34.11)$ is because of unmasking of a homozygous transition mutation in the ASS1 gene, NM_000050.4:c.571G > A (p.(Glu191Lys)). The father was identified to be a heterozygous carrier of the c.571G $>$ A mutation. Segmental isoUPD9pat gave rise to homozygosity for this mutation.

Case 2

Microarray analysis in case 2 showed two additional copies (triplication) of a 2-Mb segment at 22q12.1-q12.2. The SNP data revealed homozygosity for $22 \mathrm{q}$ immediately distal to the triplicated segment (22q12.2-q13.33; 21.2 Mb) (ISCN: $\operatorname{arr}[\mathrm{hg} 19] 22 \mathrm{q} 12.1 \mathrm{q} 12.2$ $(27781546-29782433) \times 4,22 q 12.2 q 13.33(29793640-51010112) \times$ $2 \mathrm{hmz}$ ) (Figure 2a). Genotype analysis for this case and both her parents confirmed that the isodisomic segment was maternal in origin. As described in the results for case 1 above, the allele peak pattern for the triplicated segment had only three values (tracks; AA/AA, $\mathrm{AB} / \mathrm{AB}$ or $\mathrm{AA} / \mathrm{BB}$ and $\mathrm{BB} / \mathrm{BB}$ ) strongly indicative of two copies of the $22 q 12.1-q 12.2$ segment being of maternal origin and another two copies of paternal origin (Figure $2 \mathrm{a}$ ).

Karyotype and FISH analyses. As shown in Figure 2b, dual-color FISH analysis (utilizing two BAC clones at the two opposing ends of the triplicated segment) was uninformative with regards to the arrangement of the segmental gain (Figure 2b). As expected, owing to the size of the triplication and relative small size of acrocentric chromosome 22, information other than an interstitial triplication could not be derived in this case. Extended FISH analysis ruled out mosaicism. 

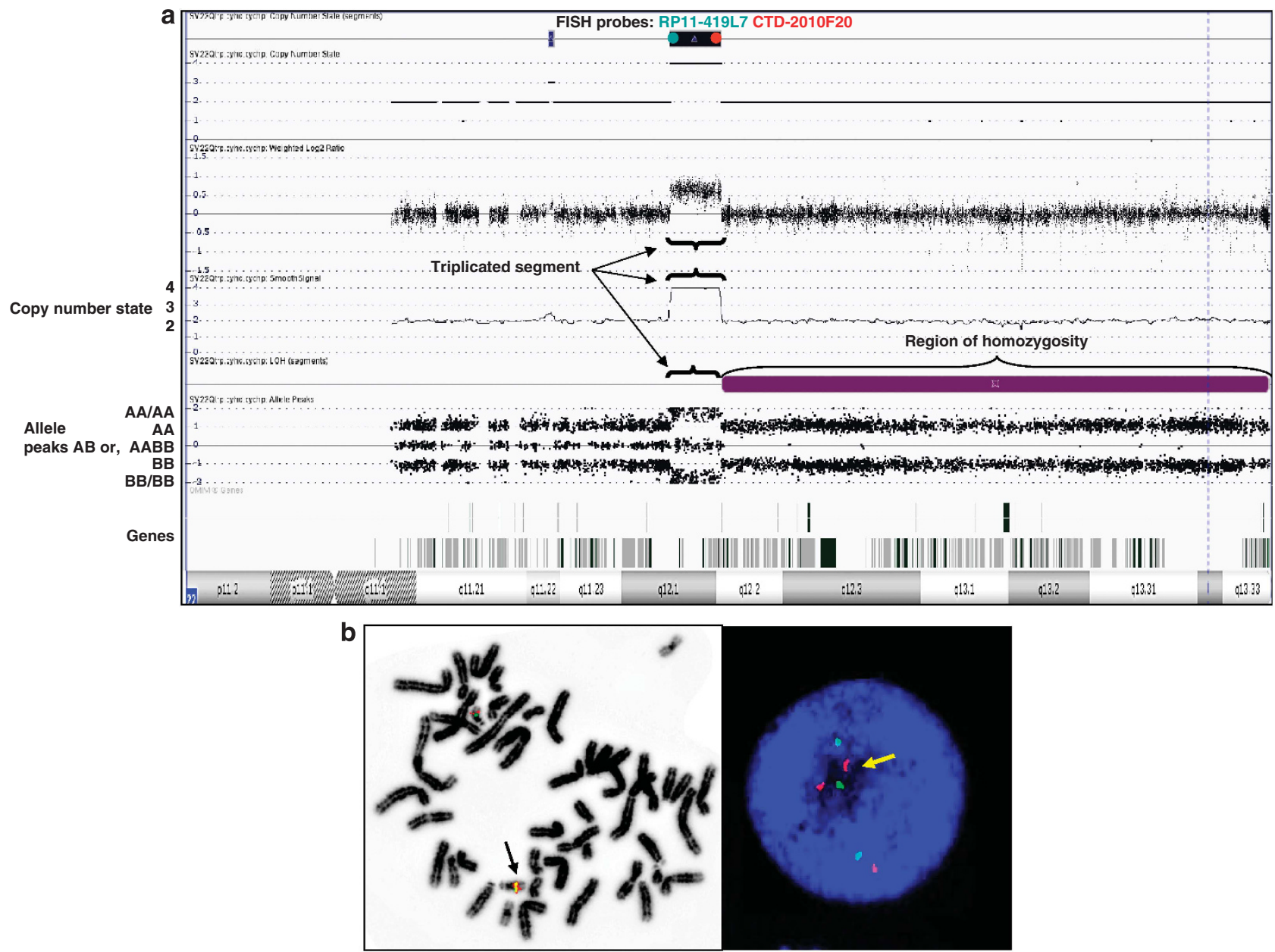

Figure 2 Panel a, case 2. Data represented for case 2 in the same order as case 1 above. Interstitial triplication coupled with distal isodisomy is highlighted. Panel b, FISH analysis with two differentially labeled probes do not differentiate the orientation of the segment within the triplication (location of BAC clones used as FISH probes is schematically represented as red and green ovals within the triplicated segment (CTD-2010F20, red; RP11-419L7, green; GRCh37/hg19 coordinates described in Patients and methods section). Black and yellow arrows in the metaphase and interphase image, respectively, indicate the abnormal chromosome 22.

The triplicated segment includes 11 OMIM annotated genes, 4 of which have been associated with a clinical phenotype (Supplementary Table 2). None of the nine OMIM listed disorders were considered clinically compatible with the patient's phenotype. The distal homozygous segment includes 215 OMIM annotated genes (44 associated with disorders).

\section{Parental studies}

Parental chromosome and microarray analyses were normal in both cases. The large number of informative SNPs within the isodisomic segments allowed genotype evaluation of patient and parents in both cases; in case 1 SNP genotype analysis revealed the segmental isodisomy was of paternal origin (Supplementary Table 3). The father in case 1 was identified as a heterozygous carrier of the c.571G $>A$ (p.(Glu191Lys)) mutation. The segmental isodisomy in case 2 was maternal in origin (Supplementary Table 4).

\section{DISCUSSION}

Nonrecurrent CCRs provide important clues toward understanding the mechanism of origin for many genomic rearrangements. Significant insights into the understanding of the phenotypic impact of such genomic rearrangements have been derived from identification of rare patients harboring them such as those reported here. The cases presented here harboring concurrent $\mathrm{CNV}$ and segmental isoUPD with a shared breakpoint within the same chromosome arm represent a potential novel disease mechanism that may influence a clinical phenotype by virtue of copy number alterations of dosage-sensitive genes, unmasking of imprinted genes or recessive mutations or a combination of both.

Although the triplicated segment in each of the cases involved a number of genes, there is no evidence that suggests these genes to be disease causing in a dosage-sensitive manner, specifically by copy number gains. However, in case 1 a clinical diagnosis of citrullinemia was molecularly confirmed. Segmental isoUPD from homozygosity for the $9 \mathrm{q} 21.33 \mathrm{q} 34.3$ segment led to the unmasking of a homozygous transition c.571G > A mutation in the ASS1 gene. This mutation was confirmed to have been inherited from a heterozygous carrier parent (father) and has been reported as pathogenic in two individual reports. ${ }^{19,20}$ Genotype-phenotype correlation in case 2 is less clear. A search of the DECIPHER database (Database of Chromosomal Imbalance and Phenotype in Humans Using Ensembl Resources) did not reveal cases with copy number gains relatively similar in size to the two cases presented here. ${ }^{21}$ One isolated report of a case with a de novo duplication of the 22q12 interval (partially overlaps the 
triplicated segment in current case 2) supposedly resulted in craniofacial abnormalities, mild intellectual adisabilities. ${ }^{22}$

These unique cases provide a new opportunity to test and understand possible mechanisms. NAHR and nonhomologous end-joining mechanisms provide a mechanistic basis for a significant fraction of recurrent and nonrecurrent microdeletions and duplications, particularly in those cases where breakpoint junctions have been sequenced and low copy repeats identified (in cases because of NAHR). ${ }^{23-25}$ More recently, nonhomologous repair mechanisms leading to copy number variations that are replication based have been proposed to explain the origin of rare and complex structural abnormalities. These replicative mechanisms are dependent upon microhomologies (short 2-15-bp stretches) and are exquisitely integrated into the DNA replication/repair machinery. These include the MMBIR and FoSTeS mechanisms, respectively. ${ }^{11,18,26}$

One of the significant predictions made from the MMBIR models is that, in addition to inducing the formation of segmental deletions and duplications, extensive loss of heterozygosity (or regions of allelic homozygosity) is likely to occur distal to the copy number alteration under certain circumstances. ${ }^{11,26}$ The MMBIR mechanism rests upon the RecA/RAD51-dependent BIR repair system that has evolved to fix single double-strand ends, where the replication fork stalls and collapses. The system has been elegantly dealt with in great detail by Hastings et $a l,{ }^{11}$ and the proposed stepwise evolution of a CNV has been hypothesized. ${ }^{11}$ Steps essential to the model include strand invasion by a single broken double-stranded segment (initiating event) into a replication fork of the sister chromatid or a chromatid of the homologous chromosome. Invasion and annealing of this broken strand in front of or behind the position of the fork is expected to lead to a deletion or duplication, respectively; in addition, if this cycle is repeated in tandem for two or more cycles, triplication or more is expected to result. Significantly, invasion and annealing into the homologous chromosome (instead of the sister chromatid) followed by completion of the replication cycle may result in extensive loss of heterozygosity distal to the duplication, triplication or deletion (Figure 3). ${ }^{11,26}$ The most important clue in support of the likely mechanism in the two cases presented here is provided by the SNP allele intensity data plotted along with copy number data. As mentioned in the results section, the allele data for the trisomic segment (three peaks $\mathrm{AA} / \mathrm{AA}, \mathrm{AB} / \mathrm{AB}$ and $\mathrm{BB} / \mathrm{BB}$ ) show the presence of the $A B / A B$ or $A A / B B$ track that is only possible if the triplicated segment (four copies of the segment genome wide) is biparental in origin. Second, with the knowledge of the parental origin of the distal isodisomic segment, it is prudent to presume that the triplication is harbored on the opposite parental chromosome 9 or 22, respectively. As we have described in detail for the first case (Results section and Figure 3), a comprehensive analysis of all the cytogenetic, microarray, genotype and molecular data suggest that the MMBIR mechanism is one of the very possible mechanisms. It is reasonable for us to suggest that replication fork collapse (at $9 \mathrm{q} 21.33$ and $22 \mathrm{q} 12.2$, respectively) leads to sister chromatid strand invasion and annealing and chain elongation for the segment in the opposing direction (Figures 3a-d); a second round of the same in the direct orientation occurs on the other parental homolog (Figure 3e), and then replication is completed with the entire segment distal to the triplicated segment being captured from a single homolog (Figure 3f). The outcome is a segmental triplication followed by segmental or distal uniparental isodisomy. For the first case, a total of three copies of the $9 \mathrm{q}$ proximal segment (two maternal and one paternal) connected to a paternal $9 q$ distal segment were identified on the hybrid maternal/paternal chromosome. The other chromosome 9 is normal and of paternal a

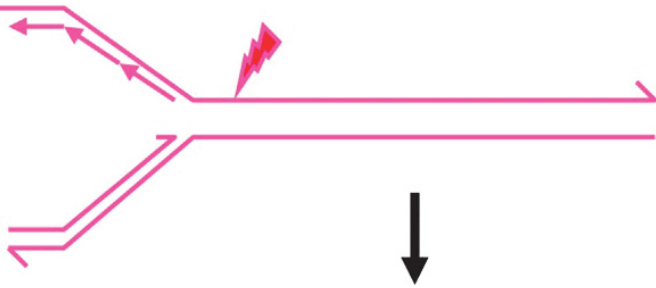

b

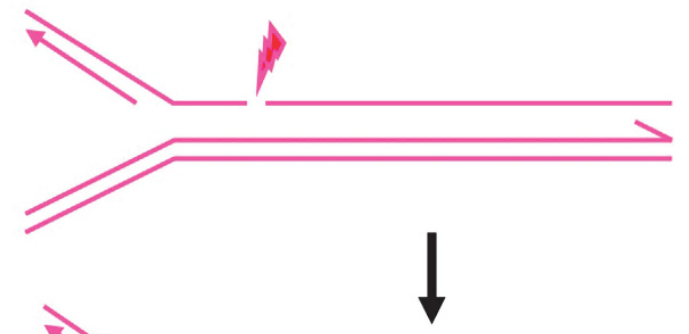

C

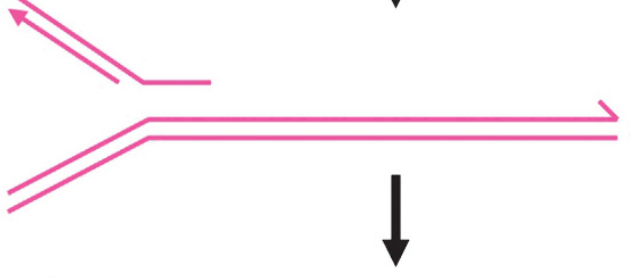

d

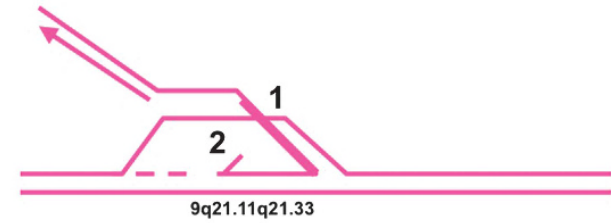

e
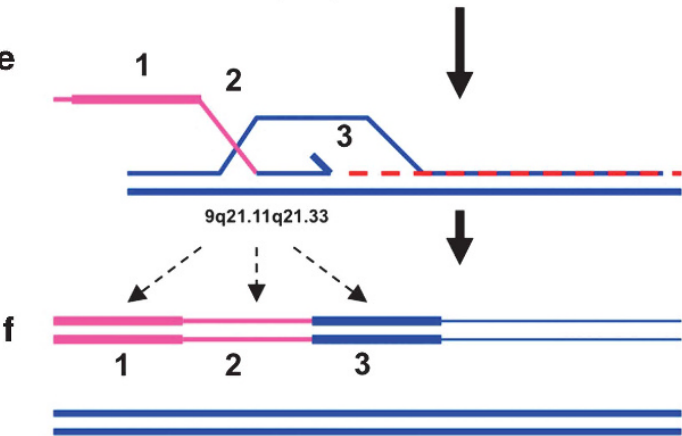

Figure $3 \mathrm{~A}$ model for MMBIR mechanism to explain the rearrangement in case 1 , and likely applicable also for case 2. (a-c) A single-ended break on one arm of replication fork at 9q21.33, the distal end of triplicated segment (9q21.11q21.33) on the maternal homolog and subsequent fork collapse. (d) Invasion of the sister chromatid at 9q21.33 in an inverted orientation and strand elongation upto proximal 9q12 (9qh). (e) Second round of invasion into paternal chr 9 homolog at $9 q 12$ and strand elongation and completion of replication. (f) Results in three copies of the 9q21.11-q21.33 segment on the maternal chromosome 9, the first two segments of maternal origin and the third of paternal origin, followed by isodisomy for the 9q21.33-qter segment of paternal origin. The segments denoted 1, 2 and 3 indicate each of the triplicated segment and parental origin (pink: maternal; blue: paternal).

origin. These two cases therefore provide strong evidence in support of the predictions made by the MMBIR models. As a post-fertilization mitotic event, it is reasonable to explore possible mosaicism; however, diligent examination of chromosome and FISH data reflected an extremely low likelihood for mosaicism. Since a second tissue was unavailable for examination if either case, tissue-specific mosaicism may not be ruled out with absolute certainty. 
A recombination-based mechanism that results in both copy number alterations and copy neutral abnormalities simultaneously cannot be excluded. A recent report by Fujita et al suggests one of two mechanisms resulting in the described complex abnormality; the possibility of two sequential post-fertilization mitotic U-type exchanges between sister chromatids and then a chromatid of the other homolog with a resulting segmental uniparental isodisomy. A second possibility is a two-step event with a sister chromatid exchange during maternal meiosis followed, as an early post-fertilization mitotic event, by a second exchange with the paternal homolog with end result being a segmental triplication of bi-parental origin and segmental isoUPD. An earlier report by Schinzel et al, ${ }^{27}$ describing a case with an intrachromosomal triplication of the 15q11-q13 region and of maternal origin, raised the possibility of an unstable abnormal intermediate (inv $\operatorname{dup}(15))$. As copy neutral loss of heterozygosity distal to the interstitial triplication was ruled out, the aberration in this case would be less likely to follow the MMBIR model for its origin. In addition, the presence of simple repeats and low copy repeat sequence elements at the breakpoints involved for each of these chromosome arms cannot be ruled out. These elements may make them susceptible to breaks or to undergo rearrangements (triplication, $\mathrm{LOH}$ ) via a recombination mechanism (Supplementary Figures 1 and 2).

As would be expected, both components of the genomic alteration can have adverse phenotypic effects. However, except for case 1 wherein we have unmasking of a deleterious homozygous mutation resulting in severe citrullinemia, the actual contribution of each of these abnormal components (CNV and UPD) is not easily predicted. This is also confounded by the fact that neither $9 \mathrm{q}$ nor $22 \mathrm{q}$ harbor imprinted genes with authentic phenotypic effects in a parent-of-origin manner. Unlike many recurrent microduplication syndromes, the phenotypic effects caused by triplication in each of these two cases, possibly quite distinct from duplication, remains uncertain. ${ }^{7}$ Finally, these cases drive the message of the profoundly significant impact of wholegenome copy-number SNP arrays in diagnostic evaluation of constitutional disorders. Second, these unique cases provide further human evidence that contribute to our understanding of complex genetic disease mechanisms and substantiate models proposed from studies in eukaryotic and prokaryotic model systems. It is reasonable to predict now that more such cases probably exist that remain unidentified because of the limited implementation of whole-genome SNP arrays. Extensive study of a larger cohort of patients harboring similar genomic rearrangements will be required to authenticate this pathogenic mechanism and derive explicit genotype-phenotype correlations.

\section{CONFLICT OF INTEREST}

The authors declare no conflict of interest.
1 Kearney HM, Kearney JB, Conlin LK: Diagnostic implications of excessive homozygosity detected by SNP-based microarrays: consanguinity, uniparental disomy, and recessive single-gene mutations. Clin Lab Med 2011; 31: 595-613, ix.

2 Papenhausen P, Schwartz S, Risheg $\mathrm{H}$ et al: UPD detection using homozygosity profiling with a SNP genotyping microarray. Am J Med Genet A 2011; 155A: 757-768.

3 Sund KL, Zimmerman SL, Thomas C et al: Regions of homozygosity identified by SNP microarray analysis aid in the diagnosis of autosomal recessive disease and incidentally detect parental blood relationships. Genet Med 2013; 15: 70-78.

4 Kotzot D: Complex and segmental uniparental disomy updated. J Med Genet 2008; 45: 545-556.

5 Liehr T, Ewers E, Hamid AB et al: Small supernumerary marker chromosomes and uniparental disomy have a story to tell. J Histochem Cytochem 2011; 59: 842-848.

6 Liehr T: Cytogenetic contribution to uniparental disomy (UPD). Mol Cytogenet 2010; 3

7 Wang JC, Fisker T, Dang L, Teshima I, Nowaczyk MJ: 4.3-Mb triplication of 4q32.1-q32.2: report of a family through two generations. Am J Med Genet $A$ 2009; 149A: 2274-2279.

8 Fujita A, Suzumura $\mathrm{H}$, Nakashima $\mathrm{M}$ et al: A unique case of de novo 5q33.3-q34 triplication with uniparental isodisomy of 5q34-qter. Am J Med Genet A 2013; 161 1904-1909.

9 Rea G, Stallings RL, Mullarkey M, McKinstry CS, McManus D, Morrison PJ: Subcortical white matter abnormalities because of previously undescribed de-novo 14q12-q13.1 triplication. Clin Dysmorphol 2013; 22: 71-72.

10 Yamamoto T, Matsuo M, Shimada S et al: De novo triplication of 11912.3 in a patient with developmental delay and distinctive facial features. Mol Cytogenet 2013; 6: 15 .

11 Hastings PJ, Lupski JR, Rosenberg SM, Ira G: Mechanisms of change in gene copy number. Nat Rev Genet 2009; 10: 551-564.

12 Carvalho CM, Ramocki MB, Pehlivan D et al: Inverted genomic segments and complex triplication rearrangements are mediated by inverted repeats in the human genome. Nat Genet 2011; 43: 1074-1081.

13 Liu P, Carvalho CM, Hastings PJ, Lupski JR: Mechanisms for recurrent and complex human genomic rearrangements. Curr Opin Genet Dev 2012; 22: 211-220.

14 Koumbaris G, Hatzisevastou-Loukidou H, Alexandrou A et al: FoSTeS, MMBIR and $\mathrm{NAHR}$ at the human proximal $\mathrm{Xp}$ region and the mechanisms of human $\mathrm{Xq}$ isochromosome formation. Hum Mol Genet 2011; 20: 1925-1936.

15 Weischenfeldt J, Symmons O, Spitz F, Korbel JO: Phenotypic impact of genomic structural variation: insights from and for human disease. Nat Rev Genet 2013; 14 125-138.

16 Madan K: Balanced complex chromosome rearrangements: reproductive aspects. A review. Am J Med Genet A 2012; 158A: 947-963.

17 Zhang F, Khajavi M, Connolly AM, Towne CF, Batish SD, Lupski JR: The DNA replication FoSTeS/MMBIR mechanism can generate genomic, genic and exonic complex rearrangements in humans. Nat Genet 2009; 41: 849-853.

18 Lee JA, Carvalho CM, Lupski JR: A DNA replication mechanism for generating nonrecurrent rearrangements associated with genomic disorders. Cell 2007; 131 1235-1247.

19 Engel K, Hohne W, Haberle J: Mutations and polymorphisms in the human argininosuccinate synthetase (ASS1) gene. Hum Mutat 2009; 30: 300-307.

$20 \mathrm{Gao} \mathrm{HZ}$, Kobayashi K. Tabata A et al: Identification of 16 novel mutations in the argininosuccinate synthetase gene and genotype-phenotype correlation in 38 classical citrullinemia patients. Hum Mutat 2003; 22: 24-34.

21 Firth HV, Richards SM, Bevan AP et al: DECIPHER: database of chromosomal imbalance and phenotype in humans using Ensembl resources. Am J Hum Genet 2009; 84: 524-533.

22 Gentile M, Wuyts W, Grittani S et al: Clinical, cytogenetic, and molecular characterization of a patient with a de novo interstitial 22q12 duplication. Am J Med Genet $A$ 2004; 127A: 186-190

23 Stankiewicz P, Shaw CJ, Dapper JD et al: Genome architecture catalyzes nonrecurrent chromosomal rearrangements. Am J Hum Genet 2003; 72: 1101-1116.

24 Lee JA, Inoue K, Cheung SW, Shaw CA, Stankiewicz P, Lupski JR: Role of genomic architecture in PLP1 duplication causing Pelizaeus-Merzbacher disease. Hum Mol Genet 2006; 15: 2250-2265.

25 Lee JA, Madrid RE, Sperle K et al: Spastic paraplegia type 2 associated with axonal neuropathy and apparent PLP1 position effect. Ann Neurol 2006; 59: 398-403.

26 Hastings PJ, Ira G, Lupski JR: A microhomology-mediated break-induced replication model for the origin of human copy number variation. PLoS Genet 2009; 5: e1000327.

27 Schinzel AA, Brecevic L, Bernasconi $F$ et al: Intrachromosomal triplication of 15q11-q13. J Med Genet 1994; 31: 798-803.

Supplementary Information accompanies this paper on European Journal of Human Genetics website (http://www.nature.com/ejhg) 